\title{
Effect of Time on Quality of Parent-Child Communication in Pediatric Cancer
}

\author{
Brittany Cowfer ${ }^{1}$, Mary Dietrich ${ }^{2}$, and Terrah Akard ${ }^{2}$ \\ ${ }^{1}$ Vanderbilt University Medical Center \\ ${ }^{2}$ Vanderbilt University School of Nursing
}

November 16, 2020

\begin{abstract}
Background A diagnosis of childhood cancer results in new parent-child communication challenges. Little is known about how communication changes over time after diagnosis or relapse. The objective of this study was to determine the effect of time since diagnosis and relapse on quality of parent-child communication. We hypothesized that there would be a positive correlation between time and quality of parent-child communication. Methods Cross-sectional study in children (7 to 17 years) with relapsed/refractory cancer and their caregivers, who spoke English, were not cognitively impaired, and had internet access. Parents were recruited through Facebook ads. Parents and children completed the Parent-Adolescent Communication Scale (PACS), a 20-item measure of communication quality, with openness and problem subscales. Spearman's Rho coefficients assessed correlations between PACS scores and time since diagnosis/relapse. Results There was a statistically significant negative correlation between parent PACS scores and time since child's cancer diagnosis (Spearman's Rho $=-0.21, \mathrm{p}=0.02$ ), indicating a tendency for overall worsening communication as time since diagnosis increased. There was a positive correlation between the parent PACS problem scores and time since diagnosis (Spearman's Rho $=+0.22, \mathrm{p}=0.01$ ), indicating more problematic communication as time since diagnosis increased. Correlations of time since relapse and PACS scores were small and not statistically significant. Conclusion Parent-child communication worsens over time following a child's cancer diagnosis with more communication problems, contrary to our hypothesis. Future studies are needed to evaluate intervention timing to best support parent-child communication beyond the new diagnosis period.
\end{abstract}

\section{Effect of Time on Quality of Parent-Child Communication in Pediatric Cancer}

Brittany A. Cowfer ${ }^{\mathrm{a}}$, MD, Mary S. Dietrich ${ }^{\mathrm{b}}$, MS, PhD, Terrah Foster Akard ${ }^{\mathrm{b}}$, PhD, RN, CPNP, FAAN

Affiliations : ${ }^{a}$ Vanderbilt University Medical Center and Monroe Carell Jr. Children's Hospital at Vanderbilt, Nashville, TN; and ${ }^{b}$ Vanderbilt University Schools of Nursing and Medicine, Nashville, TN

Address Correspondence to: Brittany Cowfer, MD, Monroe Carell Jr. Children's Hospital at Vanderbilt, Pediatric Hematology/Oncology, 2220 Pierce Ave., PRB 397, Nashville, TN 37232. Brittany.cowfer@vumc.org. Phone: 615-943-2908. Fax: 615-875-1478.

Abstract word count: 247

Main text word count: 2224

Additional files: 2 tables

Short title: Parent-Child Communication in Pediatric Cancer

Key words: Pediatric oncology, psychosocial, palliative care, parent-child communication

Abbreviations : 


\begin{tabular}{ll}
\hline NIH & National Institutes of Health \\
\hline PACS & Parent-Adolescent Communication Scale \\
IQR & Interquartile Range \\
IRB & Institutional Review Board \\
$r_{s}$ & Spearman's rho \\
\hline
\end{tabular}

\section{Abstract}

\section{Background}

A diagnosis of childhood cancer results in new parent-child communication challenges. Little is known about how communication changes over time after diagnosis or relapse. The objective of this study was to determine the effect of time since diagnosis and relapse on quality of parent-child communication. We hypothesized that there would be a positive correlation between time and quality of parent-child communication.

\section{Methods}

Cross-sectional study in children (7 to 17 years) with relapsed/refractory cancer and their caregivers, who spoke English, were not cognitively impaired, and had internet access. Parents were recruited through Facebook ads. Parents and children completed the Parent-Adolescent Communication Scale (PACS), a 20item measure of communication quality, with openness and problem subscales. Spearman's Rho coefficients assessed correlations between PACS scores and time since diagnosis/relapse.

\section{Results}

There was a statistically significant negative correlation between parent PACS scores and time since child's cancer diagnosis (Spearman's Rho $=-0.21, \mathrm{p}=0.02$ ), indicating a tendency for overall worsening communication as time since diagnosis increased. There was a positive correlation between the parent PACS problem scores and time since diagnosis (Spearman's Rho $=+0.22, \mathrm{p}=0.01$ ), indicating more problematic communication as time since diagnosis increased. Correlations of time since relapse and PACS scores were small and not statistically significant.

\section{Conclusion}

Parent-child communication worsens over time following a child's cancer diagnosis with more communication problems, contrary to our hypothesis. Future studies are needed to evaluate intervention timing to best support parent-child communication beyond the new diagnosis period.

\section{Introduction}

Approximately 16850 children and adolescents will be newly diagnosed with cancer in $2020 .{ }^{1}$ While fiveyear childhood cancer survival rates have improved to $85 \%,{ }^{1}$ cancer remains the leading cause of diseaserelated death for children in the United States, with 1730 deaths expected in $2020 .{ }^{1}$ The stress of childhood cancer often challenges parents' decision-making, time management, coping, and communication, resulting in burden on parental mental health. ${ }^{2}$ Parental distress has been shown to negatively impact parent-child communication, ${ }^{3-5}$ and ineffective and harsh communication has been correlated with psychologic problems in children with cancer. ${ }^{5,6}$

Parents have identified barriers to open and honest communication,${ }^{7}$ including desires to protect ill children from fear or other unpleasant emotions, ${ }^{8-12}$ perceptions of inappropriately burdening children, ${ }^{13,14}$ concerns that children would lose trust in them, ${ }^{11}$ fear of children's reactions, ${ }^{8,15}$ feeling too overwhelmed, ${ }^{12,13}$ and lack of confidence in communicating. ${ }^{8,13}$ Some parents have perceived that children were too young to understand, ${ }^{8}$ already knew so did not need to communicate, ${ }^{8}$ or lacked the desire to communicate. ${ }^{8,14}$ Consequently, the majority of parents of children terminally ill with cancer have avoided discussions with their child about potential death. ${ }^{8,16}$ About a quarter of those parents later regretted not telling their child, 
more commonly if they sensed their child was aware of their impending death. ${ }^{16}$ Despite ill children's frequent awareness, many children avoid communicating about death to protect their parents. ${ }^{10}$ Parents and children with cancer may also engage in mutual pretense, when they both know the difficult-to-acknowledge truth about the child's terminal prognosis but pretend as though they do not. ${ }^{8,17}$ This means of interacting is often set by parents' lack of openness in disease-related communication with their child.

Despite these challenges, communication between parents and children with cancer is crucial and has numerous benefits. The National Cancer Institute recommends that parents communicate openly and honestly with ill children about their cancer diagnosis. ${ }^{18}$ Young children value their family in helping them communicate with providers and often feel anxious when information about their disease is withheld. ${ }^{19}$ Supportive rather than invalidating parental communication has resulted in less pain and distress in children undergoing procedures, such as port access or lumbar puncture. ${ }^{20}$ In children with incurable cancer, parents have cited that communicating with their child about death allowed for parents to address fears, gave the child the opportunity to help plan their funeral, and resulted in stronger family relationships. ${ }^{8}$ Parents are motivated to communicate openly with their child when they feel it helps protect their child from fear, ${ }^{10}$ facilitates coping, ${ }^{14,21}$ or allows their child to be involved in decision-making. ${ }^{14,15}$ Many parents want to avoid lying and maintain their child's trust. ${ }^{9,12,15}$ Child-initiated conversations about their disease or death have facilitated more open parent-child communication. ${ }^{21,22}$ Studies have shown that parents may communicate because they feel it is difficult to hide information ${ }^{15}$ and are more likely to communicate with older rather than younger children. ${ }^{12,15}$

Despite these advances in research related to parent-child communication in pediatric oncology, minimal data exist for how parent-child communication changes over time throughout the illness trajectory. Specifically, initial diagnosis or relapse may be milestone timepoints that create challenges for parent-child communication. While parents may communicate more openly with older children, ${ }^{12,15}$ and children often prefer to know more about their disease over time, ${ }^{23}$ children's communication preferences may vary at different points in their illness journey, ${ }^{12,23}$ which may affect the quality and amount of information exchange between parents and children. The purpose of this study was to determine the effect of time since childhood cancer diagnosis and relapse on quality of parent-child communication. We hypothesized that there would be a positive correlation between time and quality of parent-child communication.

\section{Methods}

This study was a secondary analysis of data collected as a part of a randomized controlled trial examining the effects of a legacy intervention for children with advanced cancer and their parents. ${ }^{24}$ After obtaining Institutional Review Board approval, parents of children with cancer were recruited between 2015 and 2018 through the use of Facebook advertisements. Advertisements targeted parents in the United States who had "liked" or "followed" other childhood cancer-related Facebook pages. Advertisements included a REDCap (a secure online software for the development and analysis of research data $)^{25,26}$ survey link which described the study and asked basic screening questions, followed by a prompt for demographic and contact information. Study personnel contacted interested individuals within a week to discuss the study further. Eligibility criteria included children diagnosed with relapsed or refractory cancer aged 7 to 17 years and their primary parent caregivers (aged 18 or older) who were fluent in English, without cognitive impairment, and had internet access. The study coordinator obtained verbal parent consent and child assent. Written consent was waived per the IRB. Child-parent dyads were randomized 1:1 to a usual care or intervention group.

After consent, parents completed a demographic survey, including dates of diagnosis and relapse, if applicable. In addition to baseline (T1) and post-intervention (T2) measures of child quality of life and child and parent coping, children and parents completed the Parent-Adolescent Communication Scale (PACS ${ }^{27}$ via REDCap to assess parent-child communication. The 20-item PACS consists of two subscales, openness of and problems in communication, prompting participants to select their rating on a 5-point Likert scale from "strongly disagree" to "strongly agree." Higher scores for the openness subscale and lower scores for problem subscale suggested higher quality of communication. An overall communication score is then derived, with higher scores indicating better communication. Children were asked to complete the measure twice - once 
regarding communication with their mother and again regarding communication with their father. Parent participants completed the questionaire about communication with their child with cancer. Cross-sectional baseline demographic and PACS data from T1 only were included in this secondary analysis.

Data were analyzed using IBM SPSS Statistics Version 27. Frequency distributions were used to summarize the nominal and ordinal variables. Due to skewness, median and interquartile range (IQR) were used to summarize the continuous variables of age, time since diagnosis/relapse, and the PACS scores. Spearman's Rho coefficients assessed the correlations between the PACS scores and time since diagnosis/relapse. Statistical significance was determined by an alpha of $0.05(\mathrm{p}<0.05)$.

\section{Results}

Of 273 screened parent-child dyads, 150 dyads enrolled in the study. Dyads that completed T1 with information for time since diagnosis were included in the sample for this secondary analysis $(N=132)$. Summaries of the children and caregivers included in the analyses are shown in Table I. Children were a median 10.0 years old (IQR $=8,13)$. A majority of the children and caregivers were White ( $85 \%$ and $92 \%$ respectively). Approximately $54 \%$ of the children were female and a majority of the caregivers were female (92\%). Children were a median 4.1 years from diagnosis $(\mathrm{IQR}=1.8,6.2)$. Ninety-two $(70 \%)$ children had experienced a disease relapse and $40(30 \%)$ had refractory disease.

Summaries of the PACS scores and their correlations with time since diagnosis and relapse are shown in Table II. Most of the children completed the PACS describing communication with their mothers $(\mathrm{n}=129$ of $132,98 \%$ ), while only $61 \%(\mathrm{n}=81$ of 132$)$ completed the respective measures about their fathers.

There was a statistically significant inverse (negative) correlation between the parental overall PACS scores and the number of years since their child had been diagnosed with cancer $\left(r_{s}=-0.21, \mathrm{p}=0.02\right.$, Table 2$)$ describing a tendency for overall communication scores to decrease as the time since diagnosis increased. This overall correlation appeared to be largely explained by the positive correlation between the PACS problems scores and time since diagnosis $\left(r_{s}=+0.22, \mathrm{p}=0.01\right)$. The correlation between the parental PACS communication openness and time since diagnosis was considerably smaller than the other two and not statistically significant $\left(r_{s}=-0.11, \mathrm{p}=0.23\right)$. None of the correlations between the child reports of communication with either parent and the time since diagnosis were statistically significant $\left(r_{s}\right.$ ranged from -0.15 to $+0.16, \mathrm{p}>0.05)$. Finally, all the correlations of either parent or child PACS scores with time since relapse were quite small and not statistically significant $\left(r_{s}\right.$ ranged from -0.05 to $\left.+0.06, \mathrm{p}>0.50\right)$.

\section{Discussion}

Due to the simultaneous importance and challenge of parent-child communication in childhood cancer, research is needed to better understand how time affects the quality of communication between parents and children with cancer. Novel aspects of this study included the analyses of parent-child communication from both parent and child perspectives, while prior studies have evaluated communication based on either parent or child report. ${ }^{28,29}$ Other strengths of the study included the large sample size and inclusion of patients with relapsed/refractory cancer, a population that is typically difficult to access.

Contrary to our hypothesis, increased time since cancer diagnosis was associated with more problematic communication and worse overall communication based on parent reports. These results align with those of prior studies in pediatric cancer survivors that showed more time from diagnosis increased the likelihood of developing a struggling parent-child relationship with poor communication. ${ }^{29}$ Similarly, children newly diagnosed with non-advanced cancer have shown an increase in parent-child conflict and problematic communication over time. ${ }^{28,30}$ While more parent-child communication problems over time may be expected as children enter adolescence and strive for more autonomy, ${ }^{31}$ we conducted a post-hoc analysis that showed no statistically significant association between child age and years since diagnosis. Thus, age is an unlikely mediator of the correlation between time and problematic communication.

Our results also suggested that time from diagnosis did not impact communication openness. This is in contrast to previous findings in pediatric cancer patients, which showed a decrease in paternal openness at 
one year after diagnosis compared to time of diagnosis in children with non-advanced cancer. ${ }^{28}$ However, in studies evaluating quality of communication between adults with cancer and their adolescent children, parent-child communication openness and problems were not impacted by time since diagnosis. ${ }^{32,33}$

Interesting to note is that our results showed no statistically significant association between time from most recent relapse and quality of communication in the patients with relapsed disease. These results are congruent with a prior study, which showed that parent-child communication problems and openness were more stable in children with advanced cancer, measured in the year following relapse, than in healthy children or those with newly diagnosed non-advanced cancer. ${ }^{28}$ Ethnographic data from families who experienced childhood cancer relapse found that families strive to maintain normalcy and avoid letting cancer dominate their lives after relapse, distinct from the new-diagnosis period when the families' lives are completely centered around the child's treatment. ${ }^{34}$ This may explain why relapse appeared to be a less important milestone than diagnosis in affecting parent-child communication. While disease relapse has been associated with a closer, more involved parent-child relationship, ${ }^{29}$ with few studies evaluating communication change over time, more research is needed to understand the continued impact of relapse on parent-child communication.

Limitations of our study included potential selection bias based on the opt-in recruitment approach. The limited range of scores of the PACS may have reduced the ability to detect correlations with time. A small number of fathers participated $(\mathrm{n}=10 ; 7.7 \%)$ as primary parent caregivers; thus, the parent PACS data is biased towards maternal impressions of communication with the ill child. Only $61 \%$ of children completed the PACS about their fathers (versus 98\% about their mothers); this could be due in part to participation by single-parent families, as half of participating parents report being married. Regardless, limited father participation in pediatric research studies has been a long-standing challenge. ${ }^{35-37}$ Social media advertisements targeting fathers rather than gender-neutral "parent" strategies have recently been shown to improve recruitment in this population. ${ }^{38}$ Additional research on and implementation of paternal recruitment strategies may allow for a more balanced representation of parental perspectives in future studies.

\section{Conclusion}

With evidence that parent-child communication worsens over time with more problems in communication following the diagnosis of childhood cancer, oncologists should continue to provide families with support in communication throughout the child's illness journey rather than only in the immediate new diagnosis period. Providers should consider continued involvement of interdisciplinary teams, including psychologists, social workers, child life specialists, and palliative care providers in order to best support communication within the family over time. Furthermore, providers can encourage high-quality communication within the family by modeling open communication in the clinic, including sharing medical information with both the parent and child while eliciting questions and concerns from each, establishing the expectation for ongoing openness at home.

Future studies are needed to determine if there is an optimal time for intervention after a child's diagnosis or relapse to most effectively prevent worsening parent-child communication. Additional prospective studies evaluating parent-child communication over time from the child's cancer diagnosis through relapse may better elucidate the impact of each of these milestones on quality of communication. With many more mothers than fathers participating in this and most other studies for parents of children with cancer and other chronic childhood illnesses, further research endeavors specifically targeting fathers would also be valuable. Such research is critical to improving care and decreasing suffering for vulnerable pediatric patients and their caregivers.

Conflict of Interest Statement: The authors have no conflicts of interest to disclose.

Data Sharing Statement: The data that support the findings of this study are available upon request from the Palliative Care Research Cooperative Group.

Acknowledgements: We thank the children and parents who kindly participated in this study.

\section{References}


1. Siegel RL, Miller KD, Jemal A. Cancer statistics, 2020. CA Cancer J Clin . 2020;70(1):7-30. doi:10.3322/caac. 21590

2. Price J, Kassam-Adams N, Alderfer MA, Christofferson J, Kazak AE. Systematic Review: A Reevaluation and Update of the Integrative (Trajectory) Model of Pediatric Medical Traumatic Stress. J Pediatr Psychol . 2016;41(1):86-97. doi:10.1093/jpepsy/jsv074

3. Rodriguez EM, Murphy L, Vannatta K, et al. Maternal Coping and Depressive Symptoms as Predictors of Mother-Child Communication About a Child's Cancer. J Pediatr Psychol . 2016;41(3):329-339. doi:10.1093/jpepsy/jsv106

4. Murphy LK, Preacher KJ, Rights JD, et al. Maternal Communication in Childhood Cancer: Factor Analysis and Relation to Maternal Distress.J Pediatr Psychol . 2018;43(10):1114-1127. doi:10.1093/jpepsy/jsy054

5. Murphy L, Rodriguez E, Gerhardt C, et al. Longitudinal Associations Among Maternal Communication and Adolescent Posttraumatic Stress Symptoms after Cancer Diagnosis. Psychooncology . 2016;25(7):779786. doi:10.1002/pon.3918

6. Adduci A, Jankovic M, Strazzer S, Massimino M, Clerici C, Poggi G. Parent-child communication and psychological adjustment in children with a brain tumor. Pediatr Blood Cancer . 2012;59(2):290-294. doi:10.1002/pbc. 24165

7. Son H, Haase J, Docherty SL. Parent-Child Communication in a Childhood Cancer Context: A Literature Review. Pediatr Nurs . 45(3):13.

8. van der Geest IMM, van den Heuvel-Eibrink MM, van Vliet LM, et al. Talking about Death with Children with Incurable Cancer: Perspectives from Parents. J Pediatr . 2015;167(6):1320-1326. doi:10.1016/j.jpeds.2015.08.066

9. Wangmo T, Clercq ED, Ruhe KM, et al. Better to know than to imagine: Including children in their health care. AJOB Empir Bioeth . 2017;8(1):11-20. doi:10.1080/23294515.2016.1207724

10. Nuss SL. Redefining Parenthood: Surviving the Death of a Child.Cancer Nurs . 2014;37(1):E51-E60. doi:10.1097/NCC.0b013e3182a0da1f

11. Arruda-Colli MNF, Perina EM, Santos MA. Experiences of Brazilian children and family caregivers facing the recurrence of cancer.Eur J Oncol Nurs . 2015;19(5):458-464. doi:10.1016/j.ejon.2015.02.004

12. Coyne I, Amory A, Gibson F, Kiernan G. Information-sharing between healthcare professionals, parents and children with cancer: more than a matter of information exchange. Eur $J$ Cancer Care (Engl) . 2016;25(1):141-156. doi:10.1111/ecc.12411

13. Badarau DO, Wangmo T, Ruhe KM, et al. Parents' Challenges and Physicians' Tasks in Disclosing Cancer to Children. A Qualitative Interview Study and Reflections on Professional Duties in Pediatric Oncology. Pediatr Blood Cancer . 2015;62(12):2177-2182. doi:10.1002/pbc.25680

14. Kars MC, Grypdonck MHF, de Bock LC, van Delden JJM. The parents' ability to attend to the "voice of their child" with incurable cancer during the palliative phase. Health Psychol . 2015;34(4):446-452. doi:10.1037/hea0000166

15. Watanabe A, Nunes T, Abreu G de. Japanese parents' perception of disclosing the diagnosis of cancer to their children: Clin Child Psychol Psychiatry . Published online January 7, 2013. doi:10.1177/1359104512470599

16. Kreicbergs U, Valdimarsdóttir U, Onelöv E, Henter J-I, Steineck G. Talking about Death with Children Who Have Severe Malignant Disease. N Engl J Med . 2004;351(12):1175-1186. doi:10.1056/NEJMoa040366

17. Bluebond-Langner M. The Private Worlds of Dying Children . Princeton University Press; 1978. 
18. Children with Cancer: A Guide for Parents - National Cancer Institute. Published online September 2015. Accessed August 14, 2020. https://www.cancer.gov/publications/patient-education/guide-for-parents

19. Mant J, Kirby A, Cox KJ, Burke A. Children's experiences of being diagnosed with cancer at the early stages of treatment; an interpretive phenomenological analysis. Clin Child Psychol Psychiatry . 2019;24(1):318. doi:10.1177/1359104518788400

20. Cline RJW, Harper FWK, Penner LA, Peterson AM, Taub JW, Albrecht TL. Parent communication and child pain and distress during painful pediatric cancer treatments. Soc Sci Med . 2006;63(4):883-898. doi:10.1016/j.socscimed.2006.03.007

21. Clarke S-A, Sheppard L, Eiser C. Mothers' explanations of communicating past health and future risks to survivors of childhood cancer. Clin Child Psychol Psychiatry . 2008;13(1):157-170. doi: $10.1177 / 1359104507080997$

22. Jalmsell L, Kontio T, Stein M, Henter J-I, Kreicbergs U. On the Child's Own Initiative: Parents Communicate with Their Dying Child About Death. Death Stud . 2015;39(2):111-117. doi:10.1080/07481187.2014.913086

23. Kelly KP, Mowbray C, Pyke-Grimm K, Hinds PS. Identifying a conceptual shift in child and adolescentreported treatment decision making: "Having a say, as I need at this time." Pediatr Blood Cancer . 2017;64(4):e26262. doi:10.1002/pbc.26262

24. Akard TF, Wray S, Friedman DL, et al. Transforming a Face-to-Face Legacy Intervention to a WebBased Legacy Intervention for Children With Advanced Cancer: J Hosp Palliat Nurs . 2020;22(1):49-60. doi:10.1097/NJH.0000000000000614

25. Harris PA, Taylor R, Minor BL, et al. The REDCap consortium: Building an international community of software platform partners.J Biomed Inform . 2019;95:103208. doi:10.1016/j.jbi.2019.103208

26. Harris PA, Taylor R, Thielke R, Payne J, Gonzalez N, Conde JG. Research Electronic Data Capture (REDCap) - A metadata-driven methodology and workflow process for providing translational research informatics support. J Biomed Inform . 2009;42(2):377-381. doi:10.1016/j.jbi.2008.08.010

27. Barnes HL, Olson DH. Parent-Adolescent Communication and the Circumplex Model. Child Dev . 1985;56(2):438-447. doi:10.2307/1129732

28. Keim MC, Lehmann V, Shultz EL, et al. Parent-Child Communication and Adjustment Among Children With Advanced and Non-Advanced Cancer in the First Year Following Diagnosis or Relapse. J Pediatr Psychol . 2017;42(8):871-881. doi:10.1093/jpepsy/jsx058

29. Tillery R, Willard VW, Sharp KMH, Klages KL, Long AM, Phipps S. Impact of the parent-child relationship on psychological and social resilience in pediatric cancer patients. Psychooncology . 2020;29(2):339-346. doi: $10.1002 /$ pon. 5258

30. Katz LF, Fladeboe K, Lavi I, et al. Trajectories of Marital, Parent-Child and Sibling Conflict during Pediatric Cancer Treatment.Health Psychol Off J Div Health Psychol Am Psychol Assoc . 2018;37(8):736745. doi: $10.1037 /$ hea0000620

31. Remschmidt H. Psychosocial Milestones in Normal Puberty and Adolescence. Horm Res . 1994;41(2):1929. doi: $10.1159 / 000183955$

32. Huizinga GA, Visser A, van der Graaf WTA, Hoekstra HJ, Hoekstra-Weebers JEHM. The quality of communication between parents and adolescent children in the case of parental cancer. Ann Oncol . 2005;16(12):1956-1961. doi:10.1093/annonc/mdi395

33. Gazendam-Donofrio S, Hoekstra H, Graaf W van der, et al. Parent-child communication patterns during the first year after a parent's cancer diagnosis. Cancer . 2009;115(18):4227-4237. doi:10.1002/cncr.24502 
34. De Graves S, Aranda S. Living with hope and fear-the uncertainty of childhood cancer after relapse. Cancer Nurs . 2008;31(4):292-301. doi:10.1097/01.NCC.0000305745.41582.73

35. Phares V, Lopez E, Fields S, Kamboukos D, Duhig AM. Are Fathers Involved in Pediatric Psychology Research and Treatment? J Pediatr Psychol . 2005;30(8):631-643. doi:10.1093/jpepsy/jsi050

36. Cabrera NJ, Volling BL, Barr R. Fathers Are Parents, Too! Widening the Lens on Parenting for Children's Development. Child Dev Perspect . 2018;12(3):152-157. doi:10.1111/cdep.12275

37. Davison KK, Gicevic S, Aftosmes-Tobio A, et al. Fathers' Representation in Observational Studies on Parenting and Childhood Obesity: A Systematic Review and Content Analysis. Am J Public Health . 2016;106(11):e14-e21. doi:10.2105/AJPH.2016.303391

38. Leach LS, Bennetts SK, Giallo R, Cooklin AR. Recruiting fathers for parenting research using online advertising campaigns: Evidence from an Australian study. Child Care Health Dev . 2019;45(6):871-876. doi: $10.1111 /$ cch. 12698

\section{Hosted file}

Table 1 Sample Demographic Characteristics.pdf available at https://authorea.com/users/ 375988/articles/493155-effect-of-time-on-quality-of-parent-child-communication-inpediatric-cancer

\section{Hosted file}

Table 2 Correlations PACS.pdf available at https://authorea.com/users/375988/articles/493155effect-of-time-on-quality-of-parent-child-communication-in-pediatric-cancer 